\title{
Derandomization of Euclidean Random Walks
}

\author{
Ilia Binder* \\ Dept. of Mathematics \\ University of Toronto
}

\author{
Mark Braverman ${ }^{\dagger}$ \\ Dept. of Computer Science \\ University of Toronto
}

January 24, 2007

\begin{abstract}
We consider the problem of derandomizing random walks in the Euclidean space $\mathbb{R}^{k}$. We show that for $k=2$, and in some cases in higher dimensions, such walks can be simulated in Logspace using only poly-logarithmically many truly random bits.

As a corollary, we show that the random walk can be deterministically simulated in space $O(\log n \sqrt{\log \log n})$, where $1 / n$ is the desired precision of the simulation.
\end{abstract}

\section{Introduction}

\subsection{Space-bounded derandomization and the Dirichlet Problem on graphs}

We are interested in derandomizing some problems that can be solved by a probabilistic log-space Turing Machines. There are many ways to view a probabilistic log-space computation. By definition, a probabilistic space bounded TM has a special state where it can request a random bit. It has to use its working tape if it wants to store the bits it has seen so far. If the machine $M$ uses $S=S(n)$ cells on its working tape and queries at most $R=R(n)$ random bits, it is said to have a space/randomness complexity of $(S, R)$. By requiring that the machine terminates in $2^{O(S)}$ steps, we can make sure that for all possible random bits the machine terminates (cf. discussion in [Saks96]).

Denote the set of valid configurations of $M$ by $C_{M},\left|C_{M}\right|=2^{O(S)}$. Denote the set of accepting configurations (i.e. configurations where $M$ has terminated in the accepting state) by $C_{a c c}$, and the set of rejecting configurations by $C_{r e j}$. Denote the initial configuration by $c_{\text {init }}$. We can view the evaluation path of the machine $M$ as a random process $M_{t}$ where $M(x)_{t} \in C_{M}$ is the configuration of the computation of $M$ on $x$ at time $t$. Further, denote by $T(x)=O\left(2^{S}\right)$ the time at which the computation terminates. $T(x)$ is a random variable, and furthermore, by definition, $M(x)_{T(x)} \in C_{a c c} \cup C_{r e j}$. The "result" of the computation of $M$ on an input $x$ is its acceptance probability, $p_{a c c}(x):=P\left(M(x)_{T(x)} \in C_{a c c}\right)$. Derandomizing the machine $M$ involves giving an algorithm for computing $p_{a c c}$ within some error $\varepsilon$, with $\varepsilon$ usually being $2^{-S}$.

One way to present the computation of the probabilistic log-space machine $M$ is by considering configurations graph $G$ of the machine. The nodes of $G$ are $C_{M}$. If a configuration $U \in C_{M}$

\footnotetext{
${ }^{*}$ Partially supported by NSERC Discovery grant 5810-2004-298433

${ }^{\dagger}$ Partially supported by NSERC CGS Scholarship
} 
corresponds to the random bit querying state, then it is connected to two configurations, $V_{0}$ and $V_{1}$, one corresponding to the configuration when the requested random bit is 0 , and the other when it is 1 . If $U$ corresponds to any other configuration, then it is connected to the unique next state $V$ of $U$, unless $U \in C_{a c c} \cup C_{r e j}$. There is a natural correspondence between runs of the machine and random walks on $G$ originating at $c_{\text {init }}$ and terminating on the set $C_{a c c} \cup C_{r e j}$. The probability that the random walk terminates on $C_{a c c}$ is exactly $p_{a c c}(x)$. We can formulate this problem in slightly more general terms. The name will be explained later.

Definition 1. The Directed Dirichlet Problem DirDP is defined as follows. Input: $A$ directed graph $G$, a vertex $v_{0}$, a set $B$ of vertexes, a parameter $\varepsilon$ and a function $f: B \rightarrow \mathbb{R}$. Output: Assuming all vertexes of $V-B$ have out-degree of at least 1 , the output should be the expected value of $f(b)$, where $b$ is the first vertex in $B$ that a random walk originating at $v_{0}$ hits, computed with precision $\varepsilon$. We denote this value by $\Phi\left(v_{0}\right)$.

Here the "random walk" takes all the edges from a vertex $v$ with equal probability. Note that we do not need to worry about the representation of the function $f$, because it is not hard to see that in order to estimate $\Phi\left(v_{0}\right)$ with precision $\varepsilon$ we only need to know $f$ with precision $\Theta(\varepsilon)$.

From the discussion above, it is not hard to see that it follows that derandomizing DirDP is as hard as derandomizing space-bounded machines.

Theorem 2. The following problems are (deterministic) space-O $(S)$ reducible to each other:

- Given a probabilistic machine $M$ running in space $S=S(n)$ and randomness $R=2^{O(S)}$, and an input $x,|x|=n$, compute $p_{\text {acc }}(x)$ within an error of $\varepsilon=2^{-S}$.

- Solve the DirDP problem on a graph of size $2^{S}$ within an error of $\varepsilon=2^{-S}$.

An equivalent view on the Dirichlet problem is a global one. Suppose that instead of considering only one starting point $v_{0}$, we consider all possible starting points. Assuming that for any initial $v$ the random walk originating at $v$ eventually hits $B$ with probability 1 , we see that the function $\Phi(v)$ satisfies the following equations:

$$
\begin{cases}\Phi(v)=\frac{1}{\operatorname{deg}(v)} \cdot \sum_{(v, u) \in E} \Phi(u) & \text { for } v \notin B \\ \Phi(v)=f(v) & \text { for } v \in B\end{cases}
$$

It can be shown that under the conditions above, the equation (1) has a unique solution.

Attempts to solve DirDP, which is at least as hard as derandomizing $\operatorname{BPSPACE}(S)$, can now be restricted to different classes of graphs. One restriction would be to consider the undirected graphs, to obtain the corresponding UndirDP problem. To our knowledge, even in this case no results better than the general space $S^{3 / 2}$ derandomization [SZ99] are known. In this paper we consider an important special case of the problem, where the underlying graph has a geometric Euclidean structure.

\subsection{The classical Dirichlet Problem and its derandomization}

First, we describe the classical Dirichlet problem on $\mathbb{R}^{k}$. Given a bounded domain $\Omega$ and a continuous function on the boundary of $\Omega, f: \partial \Omega \rightarrow \mathbb{R}$, the goal is to find a function $\Phi: \Omega \rightarrow \mathbb{R}$ that satisfies:

$$
\begin{cases}\Delta \Phi(x) \equiv \sum_{i=1}^{k} \frac{\partial^{2} \Phi(x)}{\partial x_{i}^{2}}=0 & \text { for } x \in \Omega-\partial \Omega \\ \Phi(x)=f(x) & \text { for } x \in \partial \Omega\end{cases}
$$


This classical problem, dating back to the 1840s has numerous applications in Science and Engineering (see for example [CH89])

Equation (2) can be viewed as a continuous version of equation (1) because the condition $\Delta \Phi(x)=0$ on the interior of $\Omega$ can be shown to be equivalent to the following condition. For any $x$ denote by $B(x, \varepsilon)$ the (open) ball of radius $\varepsilon$ around $x$. Then for any $x \in \Omega-\partial \Omega$, and for any $\varepsilon$ such that $B(x, \varepsilon) \subset \Omega-\partial \Omega, \Phi(x)$ is equal to the average value of $\Phi(x)$ on $B(x, \varepsilon)$. Thus, just as in equation (1), we have that for any $x, \Phi(x)$ is equal to the average value of $\Phi$ on its "neighbors".
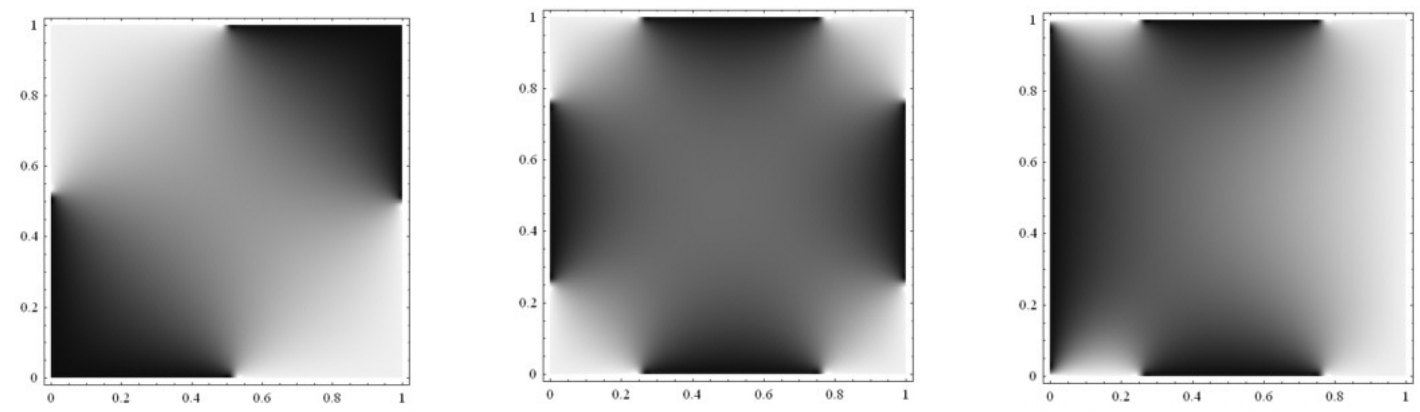

Figure 1: examples of solutions to the two-dimensional Dirichlet problem with $\Omega$ a square domain, the boundary condition $f$ is either 1 (black) or 0 (white), the color inside $\Omega$ represents the value of $\Phi$. It is equal to the probability that a Brownian motion originating at a point will hit a black segment on the boundary

As in the graph case above, Brownian motion, the continuous version of a random walk can be used to solve the Dirichlet problem. For any $x \in \Omega-\partial \Omega$, denote by $B_{t}$ the Brownian motion process originating at $x$ : $B_{0}=x$. Let the random variable $T$ be the first time $B_{t}$ hits the boundary $\partial \Omega$. Then the solution to (2) is the expected value of $f$ at $B_{T}$ :

$$
\Phi(x)=E\left[f\left(B_{T}\right)\right] .
$$

Solutions of a Dirichlet problem are illustrated on Fig. 1.

In order to approximately solve the Dirichlet problem in practice, one would need to discretize it first. This is possible under some mild conditions on the continuous problem, that will be discussed in Section 2. We define a discretize grid version of the continuous Dirichlet problem in $\mathbb{R}^{k}$.

Definition 3. Consider the subdivision of the unit cube in $\mathbb{R}^{k}$ by a grid with step $1 / n$. Let $\Omega$ be a subset of the unit cube formed by a collection of small cubes in the grid. The boundary of $\Omega$ is comprised of a finite collection $\mathcal{C}$ of $k-1$-dimensional squares. Let the boundary condition $f: \partial \Omega \rightarrow \mathbb{R}$ be given within a precision of $n^{-3}$. $f$ is continuous and linear on each of the squares of the boundary. In other words, it is specified by the values it takes on each piece $s \in \mathcal{C}$ of the boundary.

The discretize Euclidean Dirichlet problem is, given a grid point $x$ inside $\Omega$, to compute the solution $\Phi(x)$ within an error of $1 / n$. We call this problems EucDP.

The most interesting case is for $\mathbb{R}^{2}$, because of its connections to the Riemann Mapping Problem and to conformal geometry(see, for example, [Ahl53]). We almost completely derandomize the problem in this case. 
Theorem 4. The EucDP over $\mathbb{R}^{2}$ is solvable by a randomized $T M$ in space $S=O(\log n)$ using $R=O\left(\log ^{4} n\right)$ random bits.

The randomness complexity of the machine in Theorem 4 is very low compared to the $O(n)$ complexity of the naïve solution. This allows us to further derandomize it while paying only a small overhead. By using various known derandomization results, we obtain:

Corollary 5. The problem EucDP is solvable by

(a) [SZ99] a deterministic machine that uses $O(\log n \sqrt{\log \log n})$ space;

(b) [Nis94] a deterministic machine that uses $O(\log n \log \log n)$ space and runs in poly-time;

(c) [NZ96] a deterministic logspace machine that solves the problem within an error of $\frac{1}{2^{\log ^{1-\gamma} n}}$ for any $\gamma>0$.

As an application, one obtains a derandomized space-efficient algorithm for computing conformal maps.

For a simply-connected planar domain $\Omega \subsetneq \mathbb{C}$ with $w \in \Omega$. Riemann uniformization theorem states that there is unique conformal map $\psi$ of $\Omega$ onto the unit disk $\mathbb{D}$ with $\psi(w)=0, \psi^{\prime}(0)>0$. The conformal maps are used extensively in many areas, such as solving Partial Differential Equations [FS64], Hydrodynamics [LS73] Electrostatics [FLS89], and in computer tomography such as brain mapping [GWCTY04].

Theorem 6. There is an algorithm A of complexity described in Corollary 5 that computes the uniformizing map in the following sense.

Let $\Omega$ be a bounded simply-connected domain, and $w_{0} \in \Omega$. Suppose that for some $n, \partial \Omega$ is given to $A$ with precision $\frac{1}{n}$ by $O\left(n^{2}\right)$ pixels. Then $A$ computes the absolute value of the uniformizing map $\psi:(\Omega, w) \rightarrow(\mathbb{D}, 0)$ within an error of $O(1 / n)$. Furthermore, the algorithm computes the value of $\phi(w)$ with precision $1 / n$ as long as $|\psi(w)|<1-1 / n$.

The reduction of Theorem 6 to Corollary 5 is given in [BBY07].

The rest of the paper is organized as follows. In Section 2, we discuss the discretization of the continuous Dirichlet problem. The main lemma, Lemma 9, is proved in Section 3. The theorem implies Theorem 4 and, using the methods of [BBY07], Theorem 6. In Section 4, we discuss the generalization of the Theorem 4 to higher dimensions.

\section{Acknowledgements}

We would like to thank Elchanan Mossel for his helpful suggestions. The authors are also grateful to Stephen Cook for the numerous enlightening discussions.

\section{The Dirichlet problem and Euclidean random walk}

In this section we discuss the discretization of continuous Dirichlet problem. We start with the technical result about the stability of the Dirichlet problem. Informally, it states that a slight change in boundary and boundary data induces an insignificant change in the solution. 
Lemma 7. Let $\gamma_{1}, \gamma_{2}$ be two closed Jordan curves, dist $\left(\gamma_{1}, \gamma_{2}\right)<1 / n^{3}$. Let $f_{1}(t), f_{2}(t)$ be two continuous functions on $\gamma_{1}, \gamma_{2}$ correspondingly with the following continuity property: if $|x-y|<$ $1 / n^{2}, x \in \gamma_{1}, y \in \gamma_{2}$, then $\left|f_{1}(x)-f_{2}(y)\right|<1 / n$. Let $\Phi_{1}$ and $\Phi_{2}$ be the solutions of the corresponding Dirichlet problems. Let $z$ be a point inside both $\gamma_{1}$ and $\gamma_{2}$ that is at least $1 / n$-away from both curves. Then $\left|\Phi_{1}(z)-\Phi_{2}(z)\right|<2 / n$.

The lemma follows from the standard estimates in Geometric Function Theory (see, for example, [Pom92]).

The lemma allows us to approximate the solution of the Dirichlet problem on a Jordan curve $\gamma$ by approximating it using a $1 / n^{2}$-grid curve, and by approximating the boundary data by a continuous piecewise-linear function on the grid-curve (see, for example, Fig. 2). Thus the continuous Dirichlet problem, at least for domains bounded by finitely-many Jordan curves, can be solved with arbitrary precision using EucDP
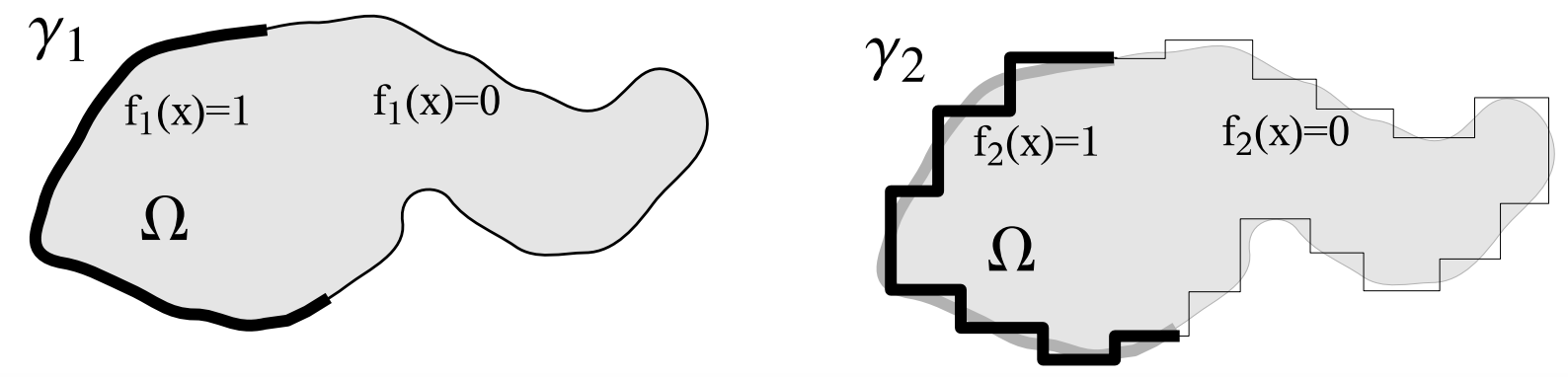

Figure 2: Discretization of the continuous Dirichlet Problem

\section{Derandomizing the Dirichlet problem}

Let us fix a bounded domain $\Omega \subset \mathbb{R}^{2}$.

Let $x \in \Omega$ with $R(x)=\operatorname{dist}(x, \partial \Omega)$. We define a stochastic jump point process $x_{t}$ inductively. At each iteration the process jumps one half of the distance to the boundary at random direction. More precisely, $x_{t}$ is defined by

$$
x_{0}=x, \quad x_{t}=x_{t-1}+1 / 2 e^{2 \pi i \theta_{t}} R\left(x_{t-1}\right) .
$$

Here $\theta_{1}, \theta_{2}, \ldots$ is a sequence of independent random variables, uniformly distributed on the interval $[0,1]$.

In the limit, this process can be used to solve Dirichlet problem. Specifically, the following Kakutani's theorem is classical.

Theorem 8. Let $f$ be a continuous function on $\partial \Omega, \Phi$ be the solution of the corresponding Dirichlet problem. A.s. $\lim _{n \rightarrow \infty} x_{n}=x_{\infty}$ exists, $x_{\infty} \in \partial \Omega$, and $\mathbf{E}\left(f\left(x_{\infty}\right)\right)=\Phi(x)$.

For the proof of the theorem, see for example [GM04], Appendix G. Let us remark that the theorem is also true for domains in $\mathbb{R}^{k}$.

Our algorithm is based on a discretized version of Theorem 8. In a discretized world, we face the following challenges. To create an efficient algorithm, we need to estimate the rate of convergence of 
the process $\left\{x_{n}\right\}$ to the boundary. We also need to take care of the rounding errors. An additional technical difficulty comes from necessity of using $x_{T}$ for certain stopping time $T$ instead of $x_{\infty}$.

To discretize the process, we fix a square grid of size $n^{-c}$ for sufficiently large constant $c$. The process will only run on points on the grid. Note that storing the coordinates of a point requires $O(\log n)$ space.

For a point $x \in \mathbb{R}^{2}$, let the snapping $S(x)$ be one of the closest points of the grid. Note that $|x-S(x)|<n^{-c}$. Let $R_{d}(x)$ be the distance from $x$ to the (discretized) boundary of $\Omega$ computed with precision of $n^{-c}$ (and requiring only $O(\log n)$ bits to store). Note that it is easy to compute in space $O(\log n)$, since the discretized boundary is just a polygon with $O\left(n^{2 c}\right)$ vertices.

Let us now discretize the process $x_{t}$. Let $X_{t}$ be the stochastic process on the grid defined by

$$
X_{0}=S(x), \quad X_{t}=S\left(X_{t-1}+1 / 2 e^{2 \pi i \theta_{t}} R_{d}\left(X_{t-1}\right)\right) .
$$

Here $\theta_{1}, \theta_{2}, \ldots$ is a sequence of independent random variables taking values $0,1 / n^{2 c}, 2 / n^{2 c}, \ldots$, $\left(n^{2 c}-1\right) / n^{2 c}$ with equal probabilities. Note that computing one iteration of $X_{t}$ requires $O(\log n)$ random bits. The process $X_{t}$ is illustrated on Fig. 3 .
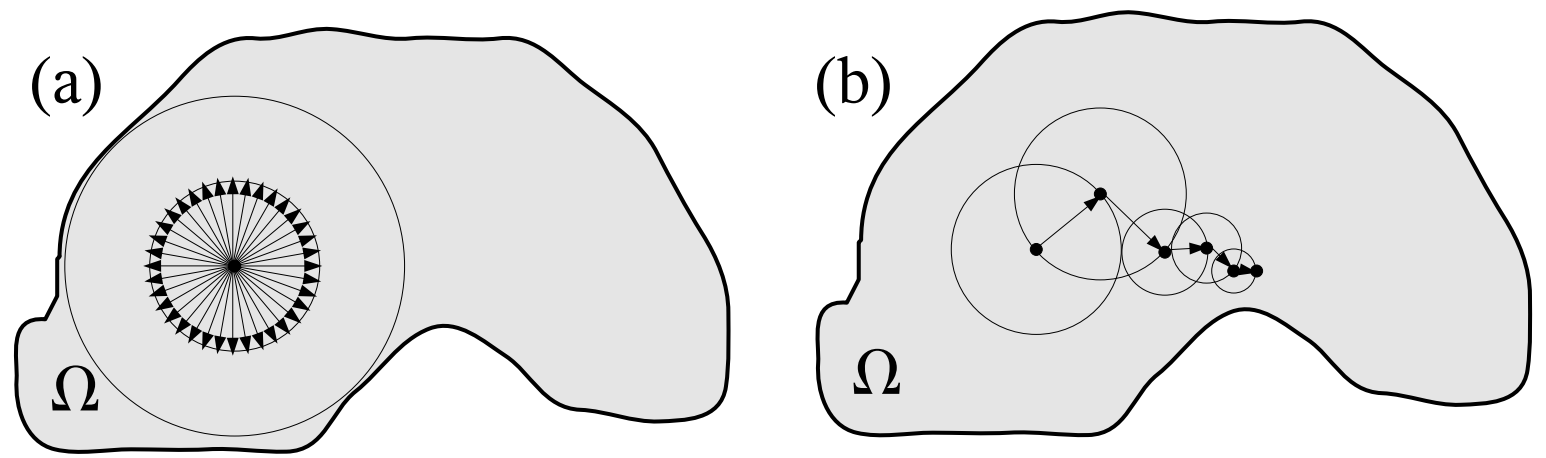

Figure 3: illustration of the process $X_{T}$. (a) one step of the process; (b) a sample path of five steps of the process

Now let us define the stopping time $T$ by $T=\min \left(\min \left\{t: R_{d}\left(X_{t}\right)<n^{-3}\right\}, B \log ^{3} n\right)$, for a sufficiently large constant $B$. In other words, we stop the process once we are sufficiently close to the boundary but never after the time $B \log ^{3} n$.

By the definition of $T$ and our discussion about the complexity of computing each jump, $X_{T}$ is computable in space $O(\log n)$ using $O\left(\log ^{4} n\right)$ random bits. We claim that the values $f\left(X_{T}\right)$ computed with precision $n^{-2}$ can be used to approximate the solution of the continuous Dirichlet problem.

Note that the boundary data $f$ from EucDP satisfies the following condition $(*)$ : If $x$ and $y$ are points in $\partial \Omega$ with $|x-y|<n^{-3}$, then $|f(x)-f(y)|<A n^{-2}$ for some constant $A$.

If $f$ is a continuous function satisfying $(*)$, then we can extend it to a grid point $x \in \Omega$ with $R_{d}(x)<n^{-3}$ by assigning $f(x)$ to be equal to $f(y)$, where $y$ is a closest to $x$ point of $\partial \Omega$. By $(*)$, it is well defined up to a $O\left(n^{-2}\right)$. For other points of the grid we take $f(x)=0$. 
Lemma 9. Let $f$ be a continuous function on $\partial \Omega$ satisfying $\left(^{*}\right)$. Let $\Phi$ be the solution of the Dirichlet problem with boundary values $f$. Let $R_{d}(x)>1 / n$. Then

$$
\left|\mathbf{E}\left[f\left(X_{T}\right)\right]-\Phi(x)\right|=O\left(n^{-2}\right) .
$$

Lemma 9 is slightly stronger than Theorem 4.

To simplify the notations, we normalize $f$ and $\Omega$ so that $\operatorname{Diameter}(\Omega)=1$ and $0 \leq f(x) \leq 1$ for all $x$.

We split the proof of the theorem into several steps.

Define the one-dimensional stochastic process $Y_{t}=\log R_{d}\left(X_{t}\right)$.

Step 1: The process $Y_{t}$ does not have a significant drift upwards. More specifically,

$$
E\left[Y_{t+1} \mid Y_{t}\right]<Y_{t}+n^{-3}
$$

Proof. Let $z$ be the point near $\partial \Omega$ realizing $R_{d}\left(X_{t}\right)$. Note also, that for any two numbers $a_{1}, a_{2}>$ $n^{-3}$ we have $\left|\log a_{1}-\log a_{2}\right|<n^{3}\left|a_{1}-a_{2}\right|$. Using the elementary fact that the function $g(x)=$ $\log |z-x|$ is harmonic on $\mathbb{R}^{2}$, we obtain

$$
\begin{aligned}
Y_{t}=g\left(X_{t}\right)=\int_{0}^{1} g\left(X_{t}+\right. & \left.\frac{R_{d}\left(X_{t}\right)}{2} e^{2 \pi i \theta}\right) d \theta> \\
& \frac{1}{n^{2 c}} \sum_{j=0}^{n^{2 c}-1} g\left(X_{t}+\frac{R_{d}\left(X_{t}\right)}{2} e^{2 \pi i \frac{j}{n^{2 c}}}\right)-\pi R_{d}\left(X_{t}\right) n^{-2 c} \cdot\left(2 / R_{d}\left(X_{t}\right)\right)>
\end{aligned}
$$

To obtain the last inequality, we approximate the integral using $n^{2 c}$ equally spaced points, the distance between adjacent points is $\pi R_{d}\left(X_{t}\right) n^{-2 c}$, and the derivative of $g$ is bounded by $2 / R_{d}\left(X_{t}\right)$. We continue the chain of inequalities by noting that the snapping operator $S$ only changes the value of $|x-z|$ by at most $n^{-c}$, and hence the value of $g(x)$ is changed by at most $n^{3-c}$,

$$
\frac{1}{n^{2 c}} \sum_{j=0}^{n^{2 c}-1} g\left(S\left(X_{t}+\frac{R_{d}\left(X_{t}\right)}{2} e^{2 \pi i \frac{j}{n^{2 c}}}\right)\right)-2 \pi n^{-2 c}-n^{3-c}>\mathbf{E}\left[Y_{t+1} \mid Y_{t}\right]-n^{-3}
$$

Step 2: For some absolute constant $\beta>0, \mathbf{E}\left[\left(Y_{t}-Y_{t-1}\right)^{2} \mid t \leq T\right]>2 \beta>0$

Proof. Let $z$ be the point near $\partial \Omega$ realizing $R_{d}\left(X_{t}\right)$. With probability at least $1 / 3, Y_{t+1}<\log \mid z-$ $X_{t+1}\left|<\log \frac{\sqrt{3}}{2}+\log \right| z-X_{t} \mid<-0.1+Y_{t}$, provided $X_{t}$ is a away from the boundary (see Fig. 4). Thus

$$
\mathbf{E}\left[\left(Y_{t}-Y_{t-1}\right)^{2} \mid t \leq T\right]>1 / 3(-0.1)^{2}>0.002>0,
$$

so we can take $\beta=0.001$.

We now define a new process $Z_{t}=Y_{t}-n^{-3} t$. Note, that by Step 1 , the process $Z_{t}=Y_{t}-n^{-3} t$ is a supermartingale. For $t>T$, we define $Z_{t}=Z_{T}$. By Doob-Meyer decomposition (see [KS91]), we can write $Z_{t}=M_{t}+I_{t}$, where $M$ is a martingale and $I_{t}$ is a decreasing adapted process. 


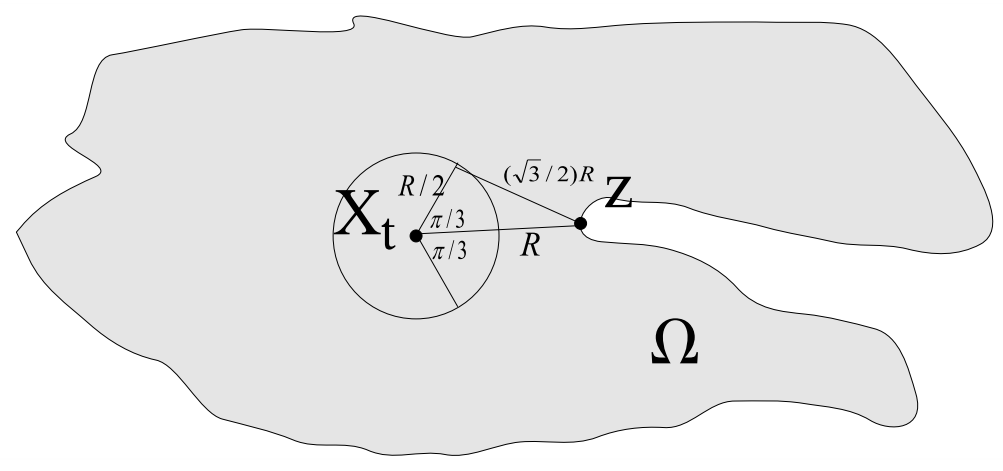

Figure 4: with probability at least $1 / 3$, the jump brings $X_{t}$ closer to $z$ by a factor of at least $\frac{\sqrt{3}}{2}$

$Z_{t} \leq Y_{t} \leq 0$, since the diameter of $\Omega$ is equal to 1 . Also, by the definition of the stopping time $T, Z_{t}>-4 \log n$. Observe that, like $Y_{t}, Z_{t}$ has definite quadratic variation at each jump. More specifically,

$$
\mathbf{E}\left[\left(Z_{t}-Z_{t-1}\right)^{2} \mid t \leq T\right]=\mathbf{E}\left[\left(Y_{t}-Y_{t-1}\right)^{2} \mid t \leq T\right]+2 n^{-3} \mathbf{E}\left[\left(Y_{t}-Y_{t-1}\right) \mid t \leq T\right]+n^{-6}>\beta
$$

provided $n$ is large.

Step 3: $\mathbf{E}\left[Z_{t-1}\left(Z_{t}-Z_{t-1}\right)\right] \geq 0$.

Proof.

$$
\begin{aligned}
\mathbf{E}\left[Z_{t-1}\left(Z_{t}-Z_{t-1}\right)\right]=\mathbf{E}\left[Z_{t-1} \mathbf{E}\left[Z_{t}-Z_{t-1} \mid Z_{t-1}\right]\right]= \\
\mathbf{E}\left[Z_{t-1} \mathbf{E}\left[M_{t}-M_{t-1} \mid Z_{t-1}\right]\right]+\mathbf{E}\left[Z_{t-1} \mathbf{E}\left[I_{t}-I_{t-1} \mid Z_{t-1}\right]\right] \geq 0 .
\end{aligned}
$$

since $\mathbf{E}\left[M_{t}-M_{t-1} \mid Z_{t-1}\right]=0, I_{t}-I_{t-1} \leq 0, Z_{t-1} \leq 0$.

Take now $T^{\prime}=C \log ^{2} n$, where $C$ is a large constant defined later.

Step 4: $\mathbf{P}\left[T \leq T^{\prime}\right]>1 / 2$.

Proof. Assume the contrary. It means that for all $t \leq T^{\prime}, \mathbf{P}[T \geq t]=1-\mathbf{P}[T<t] \geq 1 / 2$. It implies that

$$
\begin{array}{r}
\left.\mathbf{E}\left[Z_{t}^{2}\right]=\mathbf{E}\left[\left(Z_{t}-Z_{t-1}\right)+Z_{t-1}\right)^{2}\right]=\mathbf{E}\left[Z_{t-1}^{2}\right]+\mathbf{E}\left[\left(Z_{t}-Z_{t-1}\right)^{2}\right]+2 \mathbf{E}\left[Z_{t-1}\left(Z_{t}-Z_{t-1}\right)\right]= \\
\mathbf{E}\left[Z_{t-1}^{2}\right]+\mathbf{E}\left[\left(Z_{t}-Z_{t-1}\right)^{2} \mid t \leq T\right] \mathbf{P}[T \geq t]+2 \mathbf{E}\left[Z_{t-1}\left(Z_{t}-Z_{t-1}\right)\right] \geq \mathbf{E}\left[Z_{t-1}^{2}\right]+\beta / 2
\end{array}
$$

The last inequality follows from the equation (7).

It implies that $\mathbf{E}\left[Z_{T^{\prime}}^{2}\right] \geq \beta / 2 T^{\prime}=C \beta / 2 \log ^{2} n$. But $0 \geq Z_{T^{\prime}}>-4 \log n$, so $\mathbf{E}\left[Z_{T^{\prime}}^{2}\right]<16 \log ^{2} n$. So, if we take $C=32 / \beta$, we get a contradiction.

Step 5: $\mathbf{P}\left[T<B \log ^{3} n\right]>1-n^{-2}$. In other words, with high probability $X_{T}$ stops near the boundary before the time expires. 
Proof. By repeating the process from the Step 2 independently $2 \log n / \log 2$ times, we get that after $2 C \log ^{3} n / \log 2$ steps,

$$
\mathbf{P}\left[T<2 C \log ^{3} n / \log 2\right]>1-n^{-2} .
$$

It means that with probability at least $1-n^{-2}$ for some $t<2 C \log ^{3} n / \log 2, X_{t}$ is $n^{-3}$-close to $\partial \Omega$. Thus, if we take $B=2 C / \log 2$ in the definition of the stopping time $T$, we get the desired conclusion.

In the next step, we use Harnack's inequality (see, for example, [Ste93]) to estimate the distortion of the map $\Phi$ :

Lemma 10 (Harnack's inequality). Let $\Omega \subset \mathbb{R}^{k}, x, y \in \Omega, \operatorname{dist}(x, \partial \Omega)=R,\|x-y\|=r$. Let $\Psi$ be a positive harmonic function in $\Omega$. Then

$$
\left(\frac{R-r}{R+r}\right)^{k} \leq \frac{\Phi(x)}{\Phi(y)} \leq\left(\frac{R+r}{R-r}\right)^{k}
$$

Let us define two random processes we use to estimate $\left|\mathbf{E}\left[f\left(x_{T}\right)\right]-\Phi(x)\right|$ :

$$
\Phi_{t}^{+}=\Phi\left(X_{t}\right)+n^{3-c} t, \quad \Phi_{t}^{-}=\Phi\left(X_{t}\right)-n^{3-c} t
$$

Step 6: $\Phi_{t}^{+}$is a submartingale and $\Phi_{t}^{-}$is a supermartingale

Proof. The proof is very similar to the proof of Step 1. Harnack's Inequality and $0<\Phi<1$ imply that

$$
\begin{aligned}
\Phi\left(X_{t}\right)=\int_{0}^{1} \Phi\left(X_{t}+\frac{R_{d}\left(X_{t}\right)}{2} e^{2 \pi i \theta}\right) d \theta> \\
\frac{1}{n^{2 c}} \sum_{j=0}^{n^{2 c}-1} \Phi\left(X_{t}+\frac{R_{d}\left(X_{t}\right)}{2} e^{2 \pi i \frac{j}{n^{2 c}}}\right)\left(1-\pi R_{d}\left(X_{t}\right) n^{-2 c} \cdot\left(2 / R_{d}\left(X_{t}\right)\right)\right)> \\
\quad \frac{1}{n^{2 c}} \sum_{j=0}^{n^{2 c}-1} \Phi\left(S\left(X_{t}+\frac{R_{d}\left(X_{t}\right)}{2} e^{2 \pi i \frac{j}{n^{2 c}}}\right)\right)\left(1-2 \pi n^{-2 c}-n^{3-c}\right)> \\
\frac{1}{n^{2 c}} \sum_{j=0}^{n^{2 c}-1} \Phi\left(S\left(X_{t}+\frac{R_{d}\left(X_{t}\right)}{2} e^{2 \pi i \frac{j}{n^{2 c}}}\right)\right)-2 \pi n^{-2 c}-n^{3-c}>\mathbf{E}\left[\Phi\left(X_{t+1}\right)\right]-n^{-3} .
\end{aligned}
$$

It implies the first statement. The proof of the second statement is the same.

Now we are in position to prove Lemma 9.

Proof of Lemma 9. By the Submartingale Theorem (see [Dur91]), $\Phi(x)=\Phi_{0}^{+} \leq \mathbf{E}\left[\Phi_{T}^{+}\right]$. But $\Phi_{T}^{+}=\Phi\left(X_{T}\right)+n^{3-c} T \leq \Phi\left(X_{T}\right)+n^{3-c} \log ^{3} n$. Thus

$$
\begin{gathered}
\Phi(x) \leq \mathbf{E}\left[\Phi\left(X_{T}\right)\right]+n^{3-c} \log ^{3} n \leq \mathbf{E}\left[f\left(X_{T}\right)\right]+n^{3-c} \log ^{3} n+ \\
\mathbf{E}\left[\Phi\left(X_{T}\right) \mid T=B \log ^{3} n\right] \mathbf{P}\left[T=B \log ^{3} n\right]+ \\
\max _{T<B \log ^{3} n}\left(\Phi\left(X_{T}\right)-f\left(X_{T}\right)\right) \leq \mathbf{E}\left[f\left(X_{T}\right)\right]+3 L n^{-2}
\end{gathered}
$$


for large enough $L$ and $c$. Here we use the fact that the modulus of continuity of $\Phi$ is no greater than the modulus of continuity of $f$ (see [GM04]). We also use the Step 4 to obtain an estimate on $\mathbf{P}\left[T=B \log ^{3} n\right]$. Using the same reasoning for $\Phi_{t}^{-}$we get

$$
\Phi(x) \geq \mathbf{E}\left[f\left(X_{T}\right)\right]-3 L n^{-2} .
$$

Finally, by combining the two inequalities we obtain the statement of the theorem.

\section{Higher dimensions: planar-like domains}

The proposed algorithm for solving the planar Dirichlet problem does not work for general higher dimensional domains. The only obstacle to literally repeating our proof in the higher dimensions comes from the absence of the superharmonicity of the function $\log \operatorname{dist}(x, \partial \Omega$ ) (the mean-value property used in equation (4)). In other words, it is no longer true that the value of $\log \operatorname{dist}\left(X_{t}, \partial \Omega\right)$ is non-increasing on average. It turns out that the difficulty leads to an example of a domain for which our method does not converge in logarithmic number of steps, and will thus require a large number of random bits.

Let us describe an example of such a domain in $\mathbb{R}^{3}$. The domain $\Omega$ will be the unit cube with the cubes of the size $1 / n$ removed around every nonzero point of $2 / \sqrt{n}$ grid. The standard results about the random walk at $\mathbb{R}^{3}$ (see, for example, [Dur91]) imply that the random walk started at the center of the cube hits its surface before it hits the removed cubes with probability greater than $1 / 2$. Thus if the Dirichlet boundary condition $f$ is changed on the surface of the unit cube, $\Phi($ center $)$ will change significantly. On the other hand, since for any point $x \in \Omega, \operatorname{dist}(x, \partial \Omega)<1 / \sqrt{n}$, it will take $\Omega(\sqrt{n})$ steps for our process to reach the boundary of the unit cube. So our algorithm will require at least $\Omega(\sqrt{n} \log n)$ random bits.

On the other hand, for a large class of domains, which we call planar-like, our algorithm still works.

Definition 11. A domain $\Omega \subset \mathbb{R}^{k}$ is called planar-like if for every point $y \notin \Omega$ there is a $k-2$ dimensional plane containing $y$ and not intersecting $\Omega$.

Note that since $2-2=0$-dimensional planes are points, every planar domain is automatically planar-like. We can also observe that any convex domain is planar-like, since in this case for every $y \notin \Omega$ there is a $k-1$-dimensional plane containing $y$ and not intersecting $\Omega$.

It is very easy to see that the function $\log \operatorname{dist}(x, \partial \Omega)$ is superharmonic for planar-like domains (because logarithm of the distance to a $k$-2-dimensional plane is harmonic away from the plane). This allows us to slightly modify the proof of Lemma 9 (only Step 4 requires a minimal change in the constants in Harnack's principle) to obtain the following Theorem.

Theorem 12. The EucDP over $\mathbb{R}^{k}$ is solvable by a randomized $T M$ in space $S=O(\log n)$ using $R=O\left(\log ^{4} n\right)$ random bits for planar-like domains. 


\section{References}

[Ahl53] L. Ahlfors, Complex Analysis, McGraw-Hill, 1953

[BBY07] I. Binder, M. Braverman, M. Yampolsky. On computational complexity of Riemann mapping, Arkiv för Matematik, to appear. Available at www.arxiv.org/math.CV/0505617

[CH89] Courant, R. and Hilbert, D. Methods of Mathematical Physics, Vol. 1. New York: Wiley, 1989.

[Dur91] Durett, Richard Probability, Theory and Examples, Wadsworth and Brooks, 1991

[FLS89] Feynman, R. P., Leighton, R. B., and Sands, M. The Feynman Lectures on Physics, Vol. 2. Addison-Wesley, 1989.

[FS64] Fuchs, B. A. and Shabat, B. V. Functions of a Complex Variable and Some of Their Applications. Vol. 1. Pergamon Press, 1964.

[GM04] Garnett, J. B. \& Marshall, D. E. Harmonic Measure Cambridge Univ Pr, 2004, 588

[GWCTY04] Gu, X., Wang, Y., Chan, T.F., Thompson, P.M., and Yau, S.-T. Genus zero surface conformal mapping and its application to brain surface mapping, IEEE Transactions on Medical Imaging, 23, 8, pp. 949- 958, 2004

[KS91] Karatzas, I and Shreve, S.E. , Brownian Motion and Stochastic Calculus, 2nd Ed., Springer Verlag, 1991.

[LS73] Lavrentiev, M. A. and Shabat, B. V., Problems of Hydrodynamics and Their Mathematical Models, Nauka, Moscow, 1973

[Nis94] N. Nisan, $R L \subset S C$. Computational Complexity 4(1), pp. 1-11, 1994.

[NZ96] N. Nisan and D. Zuckerman, Randomness is Linear in Space. Journal of Computer and System Sciences, 52(1):43-52, 1996.

[Pom92] Pommerenke, C. Boundary Behaviour of Conformal Maps. Springer-Verlag, 1992.

[Saks96] M. Saks. Randomization and derandomization in space-bounded computation. Annual Conference on Structure in Complexity Theory, 1996.

[SZ99] M. Saks and S. Zhou. $B P_{H} S P A C E(S) \subseteq D S P A C E\left(S^{3 / 2}\right)$. Journal of Computer and System Sciences, 58 (2):376-403, 1999. 36th IEEE Symposium on the Foundations of Computer Science (FOCS), 1995.

[Ste93] Stein, E. M. Harmonic analysis: real-variable methods, orthogonality, and oscillatory integrals, Princeton University Press, 1993. 